\title{
Enhanced temporal resolution in femtosecond dynamic-grating experiments
}

\author{
Hung-Te Hsieh ${ }^{\text {a) }}$ and Demetri Psaltis \\ Department of Electrical Engineering, California Institute of Technology, Pasadena, California 91125
}

Oliver Beyer, Dominik Maxein, and Karsten Buse

Institute of Physics, University of Bonn, Wegelerstraße 8, D-53115 Bonn, Germany

Boris Sturman

Institute of Automation and Electrometry, 630090 Novosibirsk, Russia

(Received 3 December 2004; accepted 5 April 2005; published online 8 June 2005)

\begin{abstract}
Recording of gratings by interference of two pump pulses and diffraction of a third probe pulse is useful for investigating ultrafast material phenomena. We demonstrate, in theory and experiment, that the temporal resolution in such configurations does not degrade appreciably even for large angular separation between the pump pulses. Transient Kerr gratings are generated inside calcium fluoride $\left(\mathrm{CaF}_{2}\right)$ crystals by two interfering femtosecond (pump) pulses at $388 \mathrm{~nm}$ and read out by a Bragg-matched probe pulse at $776 \mathrm{~nm}$. The solution to the relevant coupled-mode equations is well corroborated by the experimental results, yielding a value of the Kerr coefficient of $\approx 4.4$ $\times 10^{-7} \mathrm{~cm}^{2} / \mathrm{GW}$ for $\mathrm{CaF}_{2}$. (C) 2005 American Institute of Physics. [DOI: 10.1063/1.1927277]
\end{abstract}

\section{INTRODUCTION}

Femtosecond pump-probe techniques have become a very powerful tool for investigating various ultrafast phenomena in materials due to the superior temporal resolution. They proved fruitful in many research areas, e.g., characterization of carrier dynamics in semiconductors, ${ }^{1,2}$ parametric up-conversion processes, ${ }^{3}$ femtosecond spectroscopy, ${ }^{4}$ and nondestructive examination of materials, ${ }^{5}$ just to name a few.

In a three-pulse pump-probe experiment, two intense, identical pump pulses overlap temporally and spatially in a nonlinear medium to induce, by interference, periodically modulated material responses within the region of intersection. A probe pulse then experiences the ensuing optical perturbations and gets diffracted if the Bragg condition is satisfied. The diffracted probe pulse is picked up by a detector; the detected signal is measured as a function of the time delay between the pump and probe pulses and gives information about the involved ultrafast mechanisms of interest. Such dynamic gratings have already been the subject of several books. 6,7

In this article, the coupled-mode equations are solved for an undepleted, Bragg-matched probe pulse in the case of a Kerr nonlinearity, which can be regarded as instantaneous compared with the pulse duration $(\approx 200 \mathrm{fs}) .^{8}$ The pumpand-probe trace thus obtained sets a limit on the achievable temporal resolution and the theoretical investigation presented in this article reveals that the temporal resolution of this pump-and-probe technique remains almost undegraded even when the angular separation between pulses is increased. This is an advantage over conventional singlepump-single-probe experiments, ${ }^{9}$ where optimal resolution is only obtained when the pulses propagate collinearly and deviation from collinearity causes the resolution to deteriorate as a result of the transverse dimensions of the pulses. ${ }^{7,10}$

${ }^{a)}$ Electronic mail: tete@caltech.edu
Experiments are conducted in calcium fluoride $\left(\mathrm{CaF}_{2}\right)$ samples to verify our theoretical prediction. Another advantage of this configuration is that the separation of the diffracted probe pulse and the pump pulses increases the signalto-noise ratio of the measurements.

\section{THEORY}

Consider the configuration depicted in Fig. 1: the spatial and temporal overlaps of two pump pulses 1 and 2 result in interference and thus the modulation of light intensity, which modifies the refractive index of the material through the optical Kerr effect

$$
n(I)=n_{0}+n_{2} I
$$

where $n_{0}$ is the usual, weak-field refractive index of the medium, $n_{2}$ is the Kerr coefficient, and $I$ is the optical intensity. The probe pulse 3 is diffracted from the resulting index grating, giving rise to the diffracted pulse 4 . The quantities

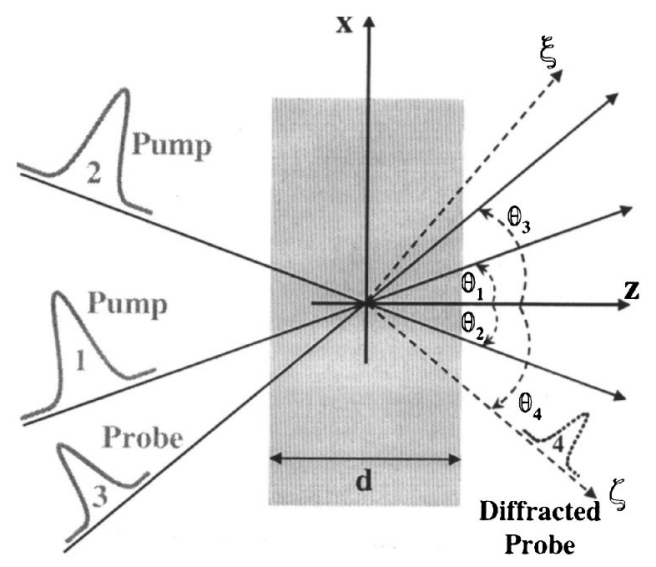

FIG. 1. Configuration of a femtosecond three-pulse pump-probe experiment in the transmission geometry; 1 and 2 are the pump (recording) pulses, while 3 and 4 are the probe and diffracted pulses. The $\zeta$ and $\xi$ axes are parallel and perpendicular to the direction of propagation of the diffracted probe pulse. 
$E_{i}(\mathbf{r}, t), \mathbf{r}=(x, y, z), \omega_{i}$, and $\mathbf{k}_{\mathbf{i}}$ represent the electric field, spatial coordinates, carrier frequency, and wave vector of pulse $i$. Here $\theta_{i}$ is the angle between $z$ axis and $\mathbf{k}_{\mathbf{i}}$. We know $\omega_{1}=\omega_{2} \equiv \omega_{p}$ and $\omega_{3}=\omega_{4} \equiv \omega_{r}$, where subscripts $p$ and $r$ denote pump and probe.

Let $\mathbf{s}_{\mathbf{i}}=\left(\sin \theta_{i}, 0, \cos \theta_{i}\right)$ be a unit vector along the direction of the wave vector $\mathbf{k}_{\mathbf{i}}$ and $k_{i}=\left|\mathbf{k}_{\mathbf{i}}\right|=\omega_{i} n_{0} / c$. We have

$$
E_{i}(\mathbf{r}, t)=\frac{1}{2} A_{i}(\mathbf{r}, t) e^{j\left(\omega_{i} t-k_{i} \mathbf{s}_{\mathbf{i}} \mathbf{r}\right)}+\text { c.c. },
$$

where $A_{i}(\mathbf{r}, t)$ is the slowly varying field amplitude of $E_{i}(\mathbf{r}, t)$. For convenience, the quantity $E_{i}(\mathbf{r}, t)$ has been normalized such that $\left|A_{i}(\mathbf{r}, t)\right|^{2}=I_{i}(\mathbf{r}, t)$, the optical intensity.

The Kerr coefficient $n_{2}$ is closely related to the thirdorder nonlinear susceptibility of the material; ${ }^{8}$ the polarization of the diffracted pulse is determined by the polarizations of pulses 1, 2, and 3 as well as by the tensorial properties of the medium. In the following subsection we resort to the scalar electromagnetic theory for its simplicity to deal with the case when all four pulses are polarized along $y$ direction. It is straightforward to extend this approach to a full tensorial notation. We will write down the equations describing the coupling between the probe and diffracted pulses owing to the concurrent pump pulses. Solutions under the assumption of undepleted probe pulse for the Bragg-matched case will be derived.

\section{A. Coupled-mode equations for the probe and diffracted pulses}

We start from the following wave equation for the light field $E_{r}(\mathbf{r}, t)=E_{3}(\mathbf{r}, t)+E_{4}(\mathbf{r}, t)$ :

$$
\nabla^{2} E_{r}(\mathbf{r}, t)-\frac{n_{r}^{2}\left(I_{p}\right)}{c^{2}} \frac{\partial^{2}}{\partial t^{2}} E_{r}(\mathbf{r}, t)=0
$$

which applies when the variation $\Delta n_{0}$ of $n_{0}(\omega)$ is small within the spectral width $\Delta \omega$ of the probe pulse and the effect of dispersion broadening is negligible for the sample thickness $d$ satisfying $d \ll c /\left(\Delta n_{0} \Delta \omega\right)$. This inequality holds for most reasonably thin samples; for example, it is satisfied in a 1 -mm-thick calcium fluoride crystal $(1 \mathrm{~mm} \ll 700 \mathrm{~mm})$ for a Gaussian pulse at $776 \mathrm{~nm}$ whose temporal full width at half maximum (FWHM) is 0.22 ps.

The refractive index $n_{r}\left(I_{p}\right)$ in Eq. (2) is specified by Eq. (1). In our formalism, the nonlinear contribution $n_{2} I_{p}$ comes from the perturbation caused by pump pulses and couples the probe pulse to the diffracted pulse when the Bragg condition

$$
\mathbf{k}_{1}-\mathbf{k}_{2}=\mathbf{k}_{3}-\mathbf{k}_{4}
$$

is satisfied. The Kerr effect transcribes the intensity pattern established by the two intense, interfering pump pulses into an instantaneous, transient index modulation

$$
n_{2} I_{p}=n_{2}\left(\left|A_{1}\right|^{2}+\left|A_{2}\right|^{2}+A_{1}^{*} A_{2} e^{j \mathbf{K} \cdot \mathbf{r}}+A_{1} A_{2}^{*} e^{-j \mathbf{K} \cdot \mathbf{r}}\right) .
$$

The spatially oscillating part on the right-hand side of Eq. (3), which can be cast as $\Delta n(\mathbf{r}, t) \cos (\mathbf{K} \cdot \mathbf{r})$, is responsible for the coupling between the (much weaker) pulses 3 and 4 . Here $\mathbf{K}=\mathbf{k}_{1}-\mathbf{k}_{2}$ is the grating vector.

In general, a set of four equations is required to describe the coupling effects between the four field amplitudes in Fig.
1 , just as in the optical four-wave mixing (FWM) configuration. In this case, however, the pump amplitudes $A_{1}$ and $A_{2}$ are barely affected by the probe amplitudes $A_{3}$ and $A_{4}$ because the pump pulses are much more intense. Moreover, it is justifiable to neglect the coupling effects between $A_{1}$ and $A_{2}$ due to a weak coupling strength. As a result, we can discard the differential equations associated with $A_{1}$ and $A_{2}$ and incorporate their effects into the term $\Delta n(\mathbf{r}, t)$.

We can write down the following coupled-mode equations for pulses 3 and 4 when the Bragg condition is satisfied, where $v_{r}$ is the group velocity of the probe pulse:

$$
\begin{aligned}
& {\left[\left(\mathbf{s}_{3} \cdot \nabla\right)+\frac{1}{v_{r}} \frac{\partial}{\partial t}\right] A_{3}=-j \frac{\pi \Delta n}{\lambda_{r}} A_{4},} \\
& {\left[\left(\mathbf{s}_{4} \cdot \nabla\right)+\frac{1}{v_{r}} \frac{\partial}{\partial t}\right] A_{4}=-j \frac{\pi \Delta n}{\lambda_{r}} A_{3} .}
\end{aligned}
$$

In arriving at the equations Eqs. (4a) and (4b) above, several approximations have been adopted:

(a) Only the third-order material nonlinearity comes into play. The second-order nonlinearity term is discarded for irrelevance; higher-order terms are also ignored because the third-order term dominates in magnitude.

(b) We ignore the terms associated with $(\Delta n)^{2}$ and keep those involving $n \Delta n$ because typically and in our experiments $\Delta n / n \lesssim 10^{-4}$.

(c) We ignore the second-order terms $\partial^{2} A_{i} / \partial t^{2}$ compared with $\omega_{r}\left(\partial A_{i} / \partial t\right)$ since the spectral bandwidth $\Delta \omega_{i}$ of $A_{i}$ is much smaller than its carrier frequency $\omega_{r}$.

(d) The longitudinal second-order derivative $\left(\mathbf{s}_{\mathbf{i}} \cdot \nabla\right)^{2} A_{i}$ is ignored compared with $k_{r}\left(\mathbf{s}_{\mathbf{i}} \cdot \nabla\right) A_{i}$ because the spatial bandwidth $\Delta k_{i}$ of $A_{i}$ is much smaller than its carrier $k_{r}$. This is the slowly varying amplitude approximation. The transverse second-order derivatives are also ignored, thanks to the next two conditions.

(e) Diffraction broadening is negligible because the Rayleigh range of the pulse is much longer than the sample thickness.

(f) The self-focusing effects experienced by the intense pump pulses can be ignored when the self-focusing distance is much larger than the sample thickness. ${ }^{8}$

The main difference between the coupled-mode equations Eqs. (4a) and (4b) for pulses and those for continuouswave $(\mathrm{cw})$ light fields is the presence of the time derivative to account for the short pulse temporal duration. Furthermore, the amplitude $A_{i}$ is a function of both $x$ and $z$ since the pulses have finite spatial extent. ${ }^{11}$

\section{B. Calculation of the diffraction efficiency}

We can obtain the amplitude of the diffracted pulse $A_{4}$ using only Eq. (4b) if we make the assumption that the probe pulse is undepleted by the transient grating; this is equivalent to the first Born approximation. ${ }^{12}$ To facilitate the solution, we convert to a retarded time frame by performing the following change of variables:

$$
\zeta=z \cos \theta_{4}+x \sin \theta_{4},
$$




$$
\begin{aligned}
& \xi=x \cos \theta_{4}-z \sin \theta_{4}, \\
& \tau=t-\frac{z}{v_{r}} \cos \theta_{4}-\frac{x}{v_{r}} \sin \theta_{4},
\end{aligned}
$$

where $\tau$ is the retarded time and $\zeta / \xi$ is the longitudinal/ transverse spatial coordinate of the diffracted pulse, as shown in Fig. 1. Let $\mathbf{r}^{\prime}=(\xi, y, \zeta)$; straightforward substitution leads us from Eq. (4b) to a much more simplified differential equation

$$
\frac{\partial A_{4}\left(\mathbf{r}^{\prime}, \tau\right)}{\partial \zeta}=-j \frac{\pi \Delta n\left(\mathbf{r}^{\prime}, \tau\right)}{\lambda_{r}} A_{3}\left(\mathbf{r}^{\prime}, \tau\right)
$$

The boundary condition $A_{4}(z=-d / 2)=0$ translates into $A_{4}(\zeta$ $\left.=-d / 2 \sec \theta_{4}+\xi \tan \theta_{4}\right)=0$ and leads to the solution at $z$ $=d / 2$

$$
A_{4}(\xi, y, z=d / 2, \tau)=\frac{-j \pi}{\lambda_{r}} \int_{-d / 2 \sec \theta_{4}+\xi \tan \theta_{4}}^{d / 2 \sec \theta_{4}+\xi \tan \theta_{4}} \Delta n A_{3} d \zeta
$$

The diffraction efficiency $\eta$ is defined as the energy ratio between the diffracted pulse and the transmitted pulse.

$$
\eta=\frac{\int_{-\infty}^{\infty} \int_{-\infty}^{\infty} \int_{-\infty}^{\infty}\left|A_{4}\left(\zeta=\frac{d}{2} \sec \theta_{4}+\xi \tan \theta_{4}\right)\right|^{2} d \xi d y d \tau}{\int_{-\infty}^{\infty} \int_{-\infty}^{\infty} \int_{-\infty}^{\infty}\left|A_{3}\left(z=\frac{d}{2}\right)\right|^{2} d x d y d t}
$$

The amplitudes of the pump and probe pulses ( $i$ $=1,2,3)$ can be represented as $A_{i}\left(\mathbf{r}^{\prime}, t\right)=\sqrt{I_{i 0}} e^{\phi_{i}\left(\mathbf{r}^{\prime}, \tau\right)}$, where $I_{i 0}$ is the peak intensity of the $i$ th pulse. Let pump and probe pulses possess temporally and spatially Gaussian profiles and be incident simultaneously onto the sample. The exponent $\phi_{i}$, which describes the free propagation of pulses 1,2 , and 3 , obeys the following relation:

$$
\begin{aligned}
\phi_{i}\left(\mathbf{r}^{\prime}, \tau\right)= & -4 \ln 2\left[\frac{\left(\tau+\frac{\zeta}{v_{r}}-\frac{\zeta \cos \alpha_{i}+\xi \sin \alpha_{i}}{v_{i}}\right)^{2}}{\tau_{i}^{2}}\right. \\
& \left.+\frac{\left(\xi \cos \alpha_{i}-\zeta \sin \alpha_{i}\right)^{2}+y^{2}}{D_{i}^{2}}\right]
\end{aligned}
$$

where $v_{i}\left(v_{1}=v_{2} \equiv v_{p}, v_{3}=v_{4} \equiv v_{r}\right)$ is the group velocity, $\tau_{i}$ and $D_{i}\left(\tau_{1}=\tau_{2} \equiv \tau_{p}, \tau_{3} \equiv \tau_{r}, D_{1}=D_{2} \equiv D_{p}, D_{3} \equiv D_{r}\right)$ are the temporal and spatial FWHM of the pulse amplitudes, and $\alpha_{i}$ is given by $\alpha_{i}=\theta_{i}-\theta_{4}$.

Now we solve for the case when the incident probe pulse is delayed by $\Delta t$ with respect to the pump pulses. The diffracted pulse amplitude $A_{4}(z=d / 2)$ becomes

$$
\begin{aligned}
& \frac{-j \pi}{\lambda_{r}} \int_{-d / 2 \sec \theta_{4}+\xi \tan \theta_{4}}^{d / 2 \sec \theta_{4}+\xi \tan \theta_{4}} 2 n_{2} \\
& \quad \times \sqrt{I_{10} I_{20} I_{30}} e^{\left[\phi_{1}\left(\mathbf{r}^{\prime}, \tau\right)+\phi_{2}\left(\mathbf{r}^{\prime}, \tau\right)+\phi_{3}\left(\mathbf{r}^{\prime}, \tau-\Delta t\right)\right]} d \zeta,
\end{aligned}
$$

where we have used $\Delta n\left(\mathbf{r}^{\prime}, \tau\right)=2 n_{2} \sqrt{I_{10} I_{20}} e^{\phi_{1}+\phi_{2}}$. Carrying out the integral and placing the result in Eq. (7), we end up

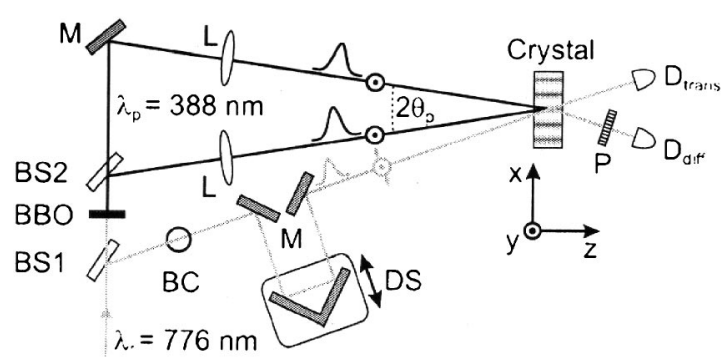

FIG. 2. Schematic illustration of the three-pulse pump-probe setup. (BC: Berek compensator, serving as half-wave plate for the probe pulse; BS: beam splitter; D: photodetector; DS: probe delay stage; L: lens; M: mirror; P: polarizer)

with an analytical expression of the diffraction efficiency, whose form lends itself to numerical evaluation.

To gain some insight into how the parameters $\theta_{i}$ and $\Delta t$ affect the diffraction efficiency, we adopt the paraxial approximation: $\sin \theta_{i} \approx \theta_{i}$ and $\cos \theta_{i} \approx 1$. By setting $\tau_{p}=\tau_{r}$ and $v_{p}=v_{r}$, we obtain after some straightforward algebraic manipulations:

$$
\eta(\Delta t) \approx \eta_{\text {peak }} \exp \left[-8 \ln 2\left(\frac{\Delta t}{\delta \tau_{r}}\right)^{2}\right] .
$$

The peak value of the diffraction efficiency $\eta_{\text {peak }}$ and the dimensionless broadening factor $\delta$ are given by

$$
\eta_{\text {peak }}=\left(\frac{2 \pi d}{\lambda_{r}}\right)^{2} \frac{n_{2}^{2} I_{10} I_{20}}{\sqrt{3+14 q}\left(1+2 D_{r}^{2} / D_{p}^{2}\right)},
$$

and

$$
\delta=\sqrt{\frac{3+14 q}{2+4 q}}
$$

where we have used $D=D_{p} D_{r} / \sqrt{D_{p}^{2}+2 D_{r}^{2}}, \theta_{1}=-\theta_{2}=\theta_{p}$, and $q=\left(D \theta_{p} / \tau_{r} v_{r}\right)^{2}$.

The function $\eta(\Delta t)$ gives us a measure of the temporal resolution of the three-pulse pump-probe through the broadening factor $\delta\left(\theta_{p}\right)$, which is the ratio between the FWHM of $\eta(\Delta t)$ and that of our probe pulse intensity profile. Although $\delta\left(\theta_{p}\right)$ is a monotonically increasing function of $\theta_{p}$, it has a rather narrow range: $1.22 \leqslant \delta<1.87$ according to Eq. (11). It is explicit that the temporal resolution is not severely affected by the angle of intersection.

The assumption of negligible difference between the pump and probe velocities, being applicable to some materials (for instance, $\mathrm{CaF}_{2}$ ), cannot be justified in the general case. The velocity difference can result in an additional broadening of $\eta(\Delta t)$.

\section{EXPERIMENTAL RESULTS AND DISCUSSION}

Our experimental setup is illustrated in Fig. 2. An axially symmetric pulse at $\lambda_{r}=776 \mathrm{~nm}$ is obtained from a Ti:sapphire amplified laser system CPA-2010, Clark-MXR, Inc. The temporal and spatial FWHM of the pulse intensity are measured to be $0.22 \mathrm{ps}$ and $3.5 \mathrm{~mm}$, respectively. Four percent of the pulse energy is tapped and serves as the probe pulse displaced by a variable delay stage. The rest of the pulse is passed through a 1 -mm-thick $\mathrm{BBO}\left(\beta-\mathrm{BaB}_{2} \mathrm{O}_{4}\right)$ 


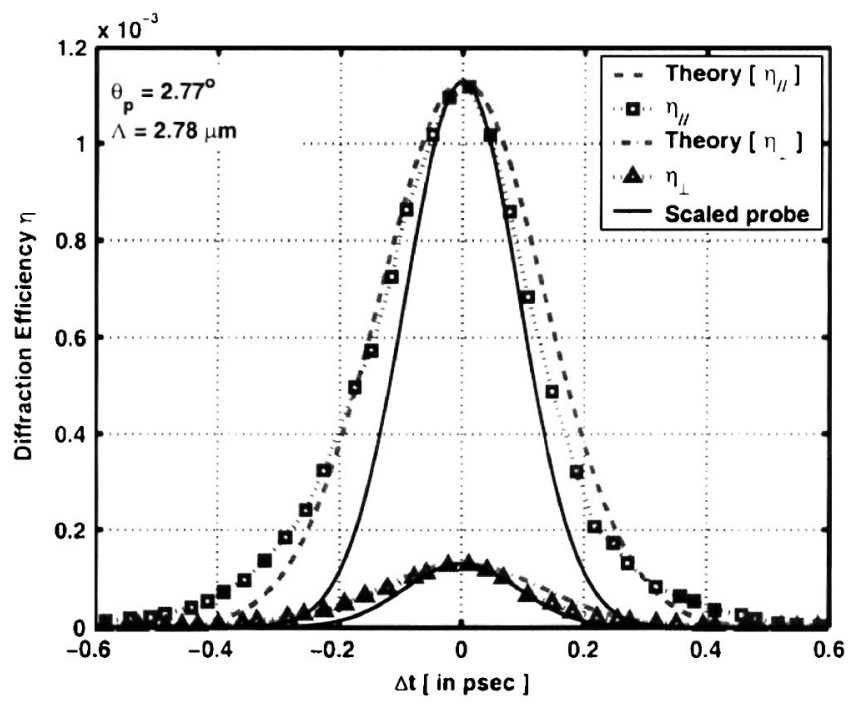

FIG. 3. (Color online). Diffraction efficiency $\eta$ vs probe delay $\Delta t$ for two different probe polarizations (parallel and perpendicular to the polarization of pump pulses, $\eta_{\|}$and $\eta_{\perp}$ ). The dashed and dash-dot lines are obtained from the theory. The solid lines are scaled probe pulse intensity profiles. $\theta_{p}$, half-angle between pump pulses. $\Lambda$, grating period.

crystal to generate a pulse at $\lambda_{p}=388 \mathrm{~nm}$, which is split into two identical pump pulses. These pump pulses are then focused down to one-fifth of their original diameter inside the 1-mm-thick calcium fluoride sample for a more pronounced nonlinear response. The angle between the pump pulses $2 \theta_{p}$ is the value inside the sample. The peak intensity of each of the pump pulses inside the sample is about $180 \mathrm{GW} / \mathrm{cm}^{2}$. The diffracted pulse is detected by a photodiode, in front of which a polarizer is used to extract the desired polarization. The optimal overlap of the pulses is then obtained by maximizing the detected diffracted pulse energy.

Calcium fluoride (chemical formula: $\mathrm{CaF}_{2}$ ) is an ionic crystal with a face-centered-cubic structure (point-group symmetry $\mathrm{m} 3 \mathrm{~m}$ ). It has a very wide band gap of about $12 \mathrm{eV}$ (Ref. 13) and a Kerr coefficient $n_{2}$ of $3 \times 10^{-7} \mathrm{~cm}^{2} / \mathrm{GW}$ around $580 \mathrm{~nm} .{ }^{8,14}$ Since the photon energy carried by our pump pulses $(3.2 \mathrm{eV})$ is far lower than the band gap, the bound electrons are responsible almost exclusively for the observed nonlinear effect. In our experiment, the third-order, nonresonant nonlinearity (or Kerr nonlinearity) is the dominant effect.

The measured diffraction efficiency for $\theta_{p}=2.77^{\circ}$ as a function of the probe delay, $\eta(\Delta t)$, is shown by the symbols in Fig. 3 for two different probe polarizations: parallel (the $\eta_{\|}$ trace) or perpendicular (the $\eta_{\perp}$ trace) to the polarization of the pump pulses. The dashed and dash-dot lines are the theoretical predictions from Eqs. (7) and (9); the appropriate group velocities used in numerical evaluations are calculated with the help of the data compiled in Ref. 15. The peak diffraction efficiency of each experimental trace is interpolated by a quadratic fit to the three highest values of $\eta(\Delta t)$. For the purpose of comparison, the scaled profiles of the incident probe intensity are also shown as solid lines. Numerical simulation shows that the trace $\eta(\Delta t)$ is symmetric and its maximum always occurs at $\Delta t=0$ when the intensity peaks of all three pulses coincide at the center of the $\mathrm{CaF}_{2}$

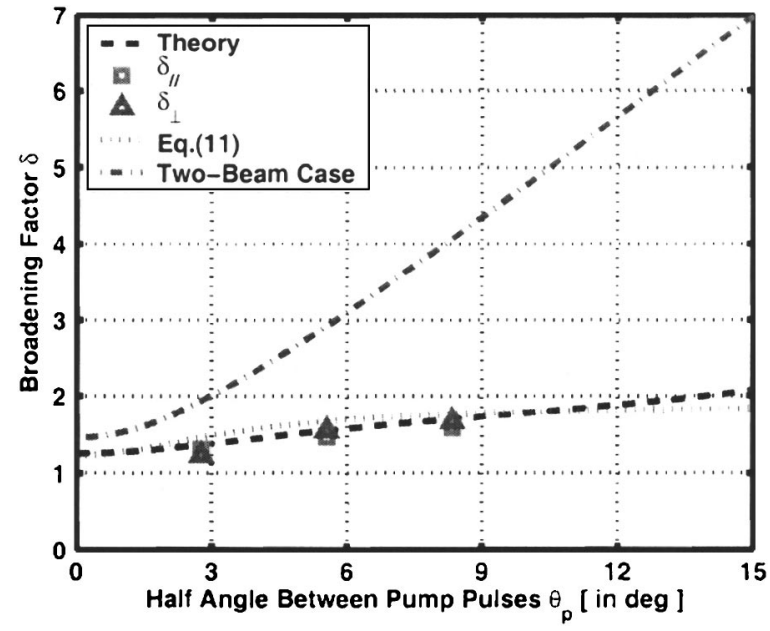

FIG. 4. (Color online). Broadening factor $\delta$ vs $\theta_{p}$, the half-angle between the pump pulses. The dashed curve is calculated numerically according to the theory; the dotted curve is plotted under paraxial approximation, using Eq. (11). The dash-dot curve, drawn here for comparison, is the temporal resolution for the single-pump-single-probe configuration.

sample which is also the origin of our coordinate system. We can see that the experimental results agree well with the theory. Moreover, the deduced value of the nonlinear refractive index $n_{2}$ is $\approx 4.4 \times 10^{-7} \mathrm{~cm}^{2} / \mathrm{GW}$, a reasonable value compared with that from the literature. ${ }^{8}$

According to the isotropic, anharmonic model of nonlinear electronic response away from material resonance, the peak diffraction efficiency of the $\eta_{\|}$trace is expected to be nine times as large as that of the $\eta_{\perp}$ trace. $^{8}$ In our experiment, this factor turns out to be $8.2 \pm 0.4$; the discrepancy can explained by experimental uncertainties and the deviation from Kleinman's symmetry. ${ }^{14}$

The broadening factor as a function of the half-angle between the pump pulses $\delta\left(\theta_{p}\right)$ is plotted in Fig. 4: the dashed curve is computed numerically according to the theory and the dotted curve is plotted using Eq. (11) under the assumption of the paraxial approximation. Experiments are carried out for three different values of $\theta_{p}$, namely, $2.77^{\circ}$, $5.55^{\circ}$, and $8.35^{\circ}$. We see that the experimental results closely track the theoretical trend and $\delta\left(\theta_{p}\right)$ almost remains constant for the experiments, as opposed to the case of the conventional two-beam pump-and-probe setup, ${ }^{7,10}$ whose broadening factor is defined based on the cross-correlation trace of the pump and probe intensities. We reproduce the two-beam broadening factor (in this case, $\theta_{p}$ is the half-angle between the pump and probe) as the dash-dot curve in Fig. 4 for the same parameters as used in our experiment.

The key to this almost undegraded temporal resolution in the three-pulse pump-probe setup lies in the concept of "composite pump," which can be explained with the help of Fig. 5. For simplicity, we consider the case when the pump pulses have a rectangular spatial intensity distribution with transverse and longitudinal dimensions $D_{p}$ and $W_{p}=v_{p} \tau_{p}$ $\left(W_{p} \approx 46 \mu \mathrm{m}\right.$ in $\mathrm{CaF}_{2}$ if $\tau_{p}=220 \mathrm{fs}$ ). The overlap of the pump pulses is shown in the upper part of the figure as a diamond-shaped region traced out by dotted lines. As the pump pulses travel and intersect, this region of overlap, the composite pump, propagates along the $z$ axis with a velocity 


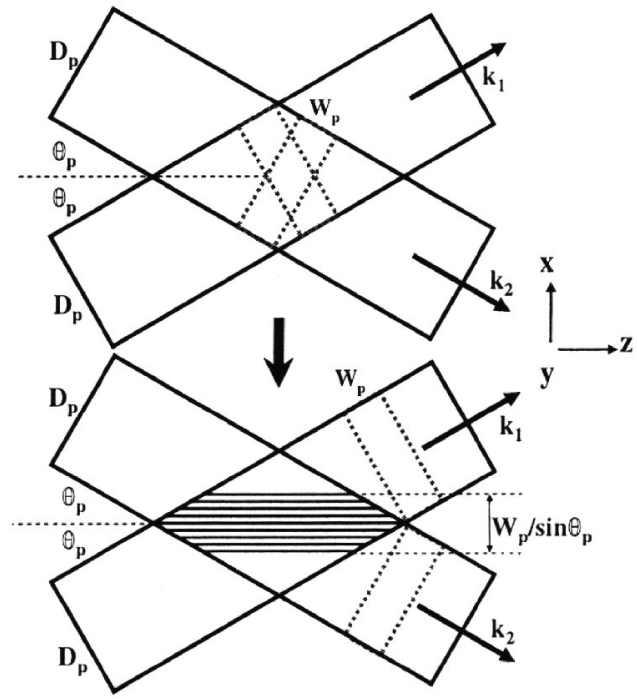

FIG. 5. The concept of the composite pump. The limited longitudinal dimension of femtosecond pulses gives rise to a striplike region of an actual overlap whose width is determined by the pulse temporal duration as well as the angle of intersection, as shown in the lower part.

$v_{c}=v_{p} / \cos \theta_{p}$ and has an effective transverse width of $D_{c}$ $=W_{p} / \sin \theta_{p}$ (shown in the lower part of the figure); this effective width becomes smaller when $\theta_{p}$ gets bigger. As evident from the expression, $D_{c}$ is independent of the spatial dimension $D_{p}$ of the pump pulses and solely determined by the pulse temporal duration $\tau_{p}$ and the angle of intersection. If we increase the angle $2 \theta_{p}$ between the pump pulses, the incident angle $\theta_{3}$ of the probe pulse must also get bigger in order to satisfy the Bragg condition.

Now we can consider the influence of the composite pump on the probe pulse just as in a two-beam case. Twobeam cross-correlation simulation suggests a broader temporal response for the increased angular intersection; on the other hand, it produces a narrower temporal response owing to a shrinking $D_{c}$. The reduction in the transverse width of the composite pump constantly counteracts the effect of an augmented probe incident angle and leaves the temporal resolution in this configuration almost unchanged.

\section{CONCLUSION}

With the assumption of an undepleted incident probe pulse, we solved the coupled-mode equations in the case of instantaneous material response for a three-pulse pumpprobe configuration where two pump pulses write a grating which is probed by the diffraction of a third, Bragg-matched pulse. The solution is well corroborated by the experiments conducted in calcium fluoride crystals. The most important implication of the experimental data obtained for different intersection angles and grating periods is that the temporal resolution in such setups is not reduced much by the noncollinearity. This result will be useful for probing gratingperiod-dependent mechanisms, such as diffusion, in materials.

\section{ACKNOWLEDGMENTS}

We thankfully acknowledge the financial support from the NSF (Engineering Research Centers Program for Neuromorphic Systems Engineering under Award No. EEC9402726, NSF-Germany Cooperative Research Grant No. INT-0233988), the Deutsche Telekom AG and the DFG (Award No. BU 913/13-1).

${ }^{1}$ A. Othonos, J. Appl. Phys. 83, 1789 (1998).

${ }^{2}$ P. C. Becker, H. L. Fragnito, C. Cruz, J. Shah, R. L. Fork, J. E. Cunningham, J. E. Henry, and C. V. Shank, Appl. Phys. Lett. 53, 2089 (1988).

${ }^{3}$ H. Mahr and M. D. Hirsch, Opt. Commun. 13, 96 (1975).

${ }^{4}$ R. T. Hayes, C. J. Walsh, and M. R. Wasielewski, J. Phys. Chem. A 108, 3253 (2004).

${ }^{5}$ P. M. Norris, A. P. Caffrey, R. J. Stevens, J. M. Klopf, J. T. McLeskey, and A. N. Smith, Rev. Sci. Instrum. 74, 400 (2003).

${ }^{6}$ H. J. Eichler, P. Günter, and D. W. Pohl, Laser-induced Dynamic Gratings (Springer, New York, 1986).

${ }^{7}$ J.-C. Diels and W. Rudolph, Ultrashort Laser Pulse and Phenomena, 1st ed. (Academic, New York, 1996).

${ }^{8}$ R. W. Boyd, Nonlinear Optics, 2nd ed. (Academic, New York, 2003).

${ }^{9}$ C. L. Thomsen, D. Madsen, S. R. Keiding, J. Thogersen, and O. Christiansen, J. Chem. Phys. 110, 3453 (1999).

${ }^{10}$ M. Ziolek, R. Naskrecki, M. Lorenc, J. Karolczak, J. Kubicki, and A. Maciejewski, Opt. Commun. 197, 467 (2001).

${ }^{11}$ L. Solymar and D. J. Cooke, Volume Holography and Volume Gratings (Academic, New York, 1981).

${ }^{12}$ M. Born and E. Wolf, Principles of Optics, 7th ed. (Cambridge, London, 1999).

${ }^{13}$ F. Gan, Y. Xu, M. Huang, W. Y. Ching, and J. G. Harrison, Phys. Rev. B 45, 8248 (1992).

${ }^{14}$ R. T. Lynch, M. D. Levenson, and N. Bloembergen, Phys. Lett. 50A, 61 (1974).

${ }^{15}$ H. H. Li, J. Phys. Chem. Ref. Data 9, 161 (1980). 